\section{The Photographic Objective}

AN interesting account of the development of the photographic lens was given recently as an address to the Physical Society by W. Taylor and H. W. Lee (Proc. Phys. Soc., May). The ordinary photographic lens has employed exclusively spherical surfaces, and successive improvements have removed one after another of the aberrations. The article describes the achromatic doublet, Lister's combination of doublets to correct coma, the Wollaston meniscus construction to correct astigmatism, and the combination of meniscus lenses to correct distortion. It next became necessary to increase the aperture of the lens, and the Petzval portrait lens appeared, followed by the 'rapid rectilinear' and the anastigmats using the new Jena glasses. H. D. Taylor in 1893 broke away from the traditional line of development and introduced an anastigmat with only three lens elements. Modern lenses are descended either from the Jena anastigmats, the Petzval lens or the H. D. Taylor lens. Diagrams of a number of modern lenses are given, including the Zeiss $f / 0.85$ lens (derived from the Petzval), various British lenses working up to $f / 1$, and special variable foeus and telephoto lenses. There is a list of references to patents.

\section{Molecular Polarisation in Solution}

IT is now known that the dipole moment of a substance measured in solution is influenced by the nature of the solvent, and some empirical rules have been proposed with the object of correcting for the solvent effect. By the examination of a number of experimental results, R. J. W. Le Fèvre (J. Chem. Soc., 773 ; 1935) concludes that the expression ${ }_{0} P_{1} / 0 P_{2}=K\left(\varepsilon_{2}+2\right) /\left(\varepsilon_{1}+2\right)$, where ${ }_{0} P_{1}$ and ${ }_{0} P_{2}$ are the orientation polarisations of a solute molecule in two media of dielectric constants $\varepsilon_{1}$ and $\varepsilon_{2}$ respectively, appears to be valid over a wide range of $\varepsilon$ values. It satisfactorily includes the polar solvents chloroform, aniline and chlorobenzene, whilst a rule previously given by Jenkins that the total polarisation is inversely proportional to $\varepsilon$ fails in this respect. A rule proposed by Müller, that ${ }_{0} P_{\text {soln }} / 0 P_{\text {gas }}=$ $1-k(\varepsilon-1)^{2}$, is found to hold only for solvents of low dielectric constant, for which $k=0.075$; it fails for chloroform $(k=0.032)$ and chlorobenzene $(k=0.035)$. The recorded data show that a nonpolar solute has much the same molecular polarisation in all solvents : only polar solutes exhibit appreciable alterations. Since the sum of the atomic and electronic polarisations of a substance is usually practically independent of its state of aggregation, these differences are wholly referable to the orientation polarisation, the variations of which are now shown to be simply related to the dielectric constant of the medium (see also p. 181 of this issue).

\section{Action of Calcium Carbide on Methyl Alcohol}

Statements occur in chemical literature to the effect that, when free from water, alcohols do not react with calcium carbide. So far as methyl alcohol is concerned, this seems to be erroneous. The results of experiments by Prof. A. Contardi and Dr. B. Ciocea (Rend. R. Ist. Lombardo Sci. e Lett., 68, parts 1-5) show that absolute methyl alcohol acts readily on powdered calcium carbide. The reaction begins even at the ordinary temperature and gives rise to calcium methoxide and acetylene, no homologues of which are formed. It is, therefore, evident that the determination of water in mixtures of hydro- carbons and alcohols by measuring the acetylene formed by their interaction with calcium carbide will not give trustworthy results unless methyl alcohol is absent. The reaction described furnishes a simple means of obtaining calcium methoxide, the preparation of which is otherwise a laborious operation.

\section{Carob Flour as a Foodstuff}

IN parts $1-5$ of vol. 68 (1935) of the Rendiconti of the Reale Istituto Lombardo di Scienze e Lettere, Prof. Adriano Valenti, of the University of Milan, gives the results of experiments in which rats and dogs were fed with carob germ flour, either raw or roasted, and in some cases boiled with water. The flour, prepared by grinding finely the germs of the carob seeds, has a sweetish taste and adheres to the palate like a gummy material. Its percentage composition was found to be: water, 8.95; proteins, $55 \cdot 30$; fats, $5 \cdot 21$; lecithin, $1 \cdot 34$; ash, $6 \cdot 70$; cellulose, $2 \cdot 32$; carbohydrates, $20 \cdot 18$. It thus appears to be richer in protein than any other food of vegetable origin. The biological experiments show, nevertheless, that it is tolerated perfectly as a food by animals. With none of the rats (26) was any ill-effect observed, and regular increase in weight occurred, particularly with young, growing animals. The rats compared well with those of a second group fed on a diet of bread and milk. Similar results were obtained with two dogs, one of which was fed, first on bread and water, then on the carob flour, and later on bread and water again; with the other dog, part of the bread in the initial and final stages was replaced by horseflesh.

\section{Lunar Heights and Topography}

Messrs. McMath, Petrie and Sawyer have developed a new technique for investigating lunar topography at the McMath-Hulbert Observatory of the University of Michigan (Publications Obs. Mich., 6, No. 8). They find that an isolated determination of the height of a lunar peak, derived from a measured shadow length, is of little significance, on account of the rugged nature of the country over which the shadow is passing. The Michigan workers investigate the variation of these relative altitudes with changing shadow lengths, and so determine the topography of the neighbouring peaks and crater walls and secure the average elevation of such points above the surrounding country. Motion picture negatives are used; these are exposed in a modified motion picture camera attached to the $10 \frac{1}{2}$ in. Cassegrain reflector. A typical set of exposure times runs from $24 \cdot 0$ sec. to $17.9 \mathrm{sec}$. with an interval of $4 \cdot 0$ sec. between exposures. After development, the negative is run through a projector which magnifies the picture to a scale of $1.15 \mathrm{~mm}$. per second of arc on the moon. A profile of a section through the crater Theophilus is given by the authors. The outer ramparts are $15,000 \mathrm{ft}$. and $14,000 \mathrm{ft}$. and the central peak $7,500 \mathrm{ft}$. above the adopted datum level, below which the floor of the crater sinks $1,000 \mathrm{ft}$. in places. This section through the crater is 65 miles from rampart to rampart. The uncertsinty is given as being $\pm 125 \mathrm{ft}$. for the elevation of a peak $16,000 \mathrm{ft}$. above the average of the terrain in its vicinity.

ERRATUM : Referring to the paragraph entitled "Sex and Cultural Status" in NATuRE of July 27, p. 149, Dr. J. D. Unwin informs us that the word "zooistic" should read "zoistic" throughout. 